\title{
Pagdukal sa mga alamat ng mga barangay sa Bayan ng Matalom, Leyte: Isang suring-tematiko
}

\author{
Gili, Victoria G \\ Matalom National High School-Matalom, Leyte, Philippines (victoria.gili@deped.gov.ph)
}

Galdo, Mark B.

Southern Leyte State University - Tomas Oppus, Philippines (mgaldo05@gmail.com)

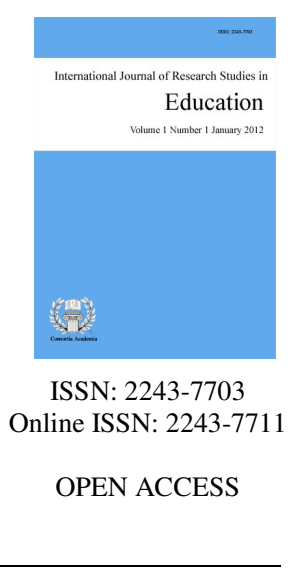

Received: 30 July 2021

Revised: 6 August 2021 DOI: $10.5861 /$ ijrse.2021.a041

Accepted: 9 August 2021

\section{Abstract}

This study aims to document and describe the culture and tradition contained in the legends in the town of Matalom, Leyte from upland (mountainous part), lowland (plains) and coastal (coastline). Using the Qualitative Research Method in the form of content analysis and historical form, the legends of fifteen (15) barangays in Matalom, Leyte were analyzed with 75 total participants. The constant comparative technique or coding method was used in the study to present the theme that was prominently contained in each location. Upon verification of the data, there was a focus group discussion with the participants. The study found that the legends of Matalom, Leyte, specifically in the fifteen barangays, are good human relations (mabuting pakikipagkapwa-tao); relying on the grace of nature (umaasa sa biyaya ng kalikasan); spiritual and environmental forces (pwersang ispirituwal at pangkalikasan); and a combination of old-fashioned beliefs and Christian beliefs (pinagsamang makalumang paniniwala at paniniwalang Kristiyano). The legends of the town of Matalom, Leyte are rich in culture and traditions that emphasize the human importance of the people of Matalomnon. From the study, a collection of legends was developed that will serve as teaching tools in $\mathrm{K}$ to 12 , specifically in the town of Matalom, Leyte.

Keywords: legend, coastal, culture, lowland, tradition, upland 


\section{Pagdukal sa mga alamat ng mga barangay sa Bayan ng Matalom, Leyte: Isang suring-tematiko}

\section{Introduksyon}

Ang panitikan ay sumasalamin sa mga paniniwala, kultura at tradisyon ng isang lugar. Sa panitikan ibinubuhos ng mga tao ang kanilang mga saloobin, nararamdaman at nakikita sa kanilang katayuan. Kung kaya, sa panitikan mababakas ang ating panlipunan at panlahing pagkakakilanlan lalong-lalo na sa mga alamat (Villafuerte, 2000). Ang alamat ay isa sa mga mahahalagang mukha ng panitikan sapagkat ito ang bumubuo ng identidad ng isang lipunan. Ang panitikang ito ay koleksyon ng mga kwentong nag-uugnay sa mga tao, lugar, hayop at mga bagay na nasa loob mismo sa konteksto ng lipunan. Sinisiyasat nito ang mga lokal na kaugalian at paniniwala na inilipat sa isang henerasyon patungo sa iba pang henerasyon na isinasalin sa pamamagitan ng pasalindila. Ang mga ito ay ekspresyon ng kultura na purong Pilipino galing sa mayamang nakaraan hanggang sa pag-usbong nito sa kasalukuyan hanggang sa hinaharap, kasaganaan, pagkakaisa at kooperasyon (Young, 2007).

Kitang-kita sa kontemporaryong panahon ang pamana ng lahing Filipino ay unti-unting nawawala. At upang hindi ito mawala, isa sa kayamanang pampanitikan ang susi sa pagbibigay-halaga sa patuloy na pagkilala ng lahing Filipino at ito ay ang alamat (Ramos, 1984). Isa ang alamat sa tatlong mahalagang pangkat ng kwentong-bayan sa panitikang Pilipino na sumasagot o nagpapaliwanag sa mga katanungang-pinagmulan, kadahilanan at iba pa. Ito ay hindi dapat nating kalimutan dahil ito ay siyang pinakamahalagang alaala sa lahing bago lumaya ay dumanas muna ng maraming kaapihan. Ito ang nagsasalaysay ng malungkot na alaala ng isang pook, ang gunitain ng isang bagay na nakapagpapadaloy ng luha at nakapagbibinhi ng dusa sa puso o kaya'y ang kadakilaan ng isang alamat upang maipabatid ang malungkot o maligayang kahapon ng kanilang lipi (Du, 2014).

Ang pag-aaral na ito ay naglalayong mapatunayan na ang mga alamat sa bayan ng Matalom, Leyte ay napapalooban ng mayamang kultura at tradisyon. Kaakibat nito, mahalagang pag-aralan ang kultura at tradisyong napapaloob sa isang alamat dahil nagsisilbi itong tulay sa pagpapayaman at pag-uunawa sa pagkakakilanlan ng isang lahi. Dala na rin sa mithiin ng K to 12 na sariwain ang mga kultura ng isang lugar kung saan napabilang ang isang mag-aaral alinsunod sa kontekswalisasyon at lokalisasyong ninanais makamit sa malawakang kamalayan sa sariling kultura bago mapag-aralan ang kultura ng iba (Lamiseria, 2016).

Ang pag-aaral na ito ay makatutulong sa paglinang ng mga lokal na panitikan sa pamamagitan ng pagsusuri ng sariling kaugalian at tradisyon, paniniwala at pamumuhay ng isang pangkat. Makatutulong ang resulta ng pag-aaral sa mga guro, sa paaralan o sa Institusyong Pang-edukasyon partikular ang Kagawaran ng Edukasyon (DepEd) bilang tugon ng programang $\mathrm{K}$ to 12 kurikulum sa kontekswalisasyon at lokalisasyon ng kurikulum. Isa rin itong ambag para sa pamahalaan, sa mga magulang sa paghubog sa mga anak sa pamamagitan ng pagtuturo ng sariling kultura, at sa mga Matalomnon na maging mulat at mapagmatyag sa kinagisnang kultura na pinagsikapang panatilihin at buhayin sa kasalukuyang pamumuhay, higit sa lahat sa mga mag-aaral na maging malawak ang kaisipan sa paghubog at pagpapayaman sa sariling kultura para maipasa pa sa mga susunod na henerasyon.

Kung kaya, nahikayat ang mananaliksik na maidokumento at mailarawan ito dahil karamihan sa mga alamat na nailimbag at nababasa sa mga modyul ng K to 12 ay mga alamat na nanggagaling sa ibang rehiyon sa bansa. Dagdag pa, tinutugon din sa pag-aaral na ito ang nilalayong kontekstwalisasyon at lokalisasyon ng $\mathrm{K}$ to 12 kurikulum sa kasalukuyan. Anumang mga alamat ang malilikom sa pag-aaral na ito ay gagamitin bilang lunsaran sa pagtuturo ng panitikang Filipino nang sa ganoon unti-unting makikilala ng mga mag-aaral ang tunay na kwento sa kanilang lugar. Kung kaya, ang layunin ng pag-aaral na ito ay maidokumento at mailarawan ang kultura at tradisyon na napapaloob sa mga alamat sa bayan ng Matalom, Leyte. 


\subsection{Teoretikal at Konseptwal na Balangkas ng Pag-aaral}

Sa pagsusuring tematiko ng mga alamat sa piling barangay ng Matalom, Leyte, ang pag-aaral na ito ay nakaangkla sa konsepto ni Panganiban at Casanova (2005) na nagsasaad na ang bawat alamat ay may taglay na kahalagahang panlipunan at pangkultural hinggil sa pinagmulan ng isang bayan o pook. Ang panitikan tulad ng alamat ay ginagamit bilang lunsaran sa pag-unawa ng kultura, magkapareho man o magkaiba sa bawat lipunan kaya ang mga mambabasa sa oras na babasahin ang isang akdang pampanitikan masasalamin nito o kakikitaan ng mga katangiang pangkultura ang mga tao sa bansang kinatawan nito (Du, 2014 at Menor, 2015). Nakikilala ang isang tao sa kulturang kanyang dala-dala kahit saan man siya magpunta dahil ito ang humuhubog sa kanyang pagkatao. May taglay na halagahang panlipunan at pangkultura ang halos lahat ng mga alamat hinggil sa pinagmulan ng isang pook o halaman. May alamat na nagsisiwalat sa paniwala't kaugalian ng lahi o pangkat ng tao.

Kaugnay nito, ang mga alamat na matatagpuan sa upland, lowland at coastal na lokasyon sa bayan ng Matalom, Leyte ang pagtutuonan ng pag-aaral. Ang kultura at tradisyon ng mga alamat ng Matalom ay makatutulong upang maging malinaw sa mga mag-aaral kung ano nga ba ang mga kaugalian ng kanilang mga ninuno na hindi na naipasa sa kanilang henerasyon at sa pamamagitan nito ay kanilang maiisip na dapat ibalik at panatilihin ang sariling pagkakailanlan. Ang pook na kinalalagyan at identidad ay may malaking kaugnayan. Patunay ito na sumasalamin ang alamat sa kultura at tradisyon ng bawat lokasyon mula upland, lowland at coastal. Sumasagisag din ito sa estado ng pamumuhat ng bawat lokasyon na kinabibilangan ng alamat na kung makikita ang litaw na litaw na kaugalian ng mga Matalomnon sa bawat barangay na sakop nito.

Tugon din ito sa hangarin ng programang $\mathrm{K}$ to 12 kurikulum na kailangang malinang ang pagpapahalagang pampanitikan ng mga mag-aaral sa kanilang partikular na lugar sa mithiing lokalisasyon at kontekswalisasyon sa kurikulum. Lalong mapalapit ang mga mag-aaral sa kanilang lugar kung ang mga kagamitang pampagtuturo na ginagamit sa pagtuturo ay malapit sa kanilang diwa at puso. Isa itong uri ng mahalagang panlunas na tumutulong sa mga mag-aaral upang makapagplano ng sari-sariling mga buhay, upang matugunan ang kanilang mga suliranin, at upang maunawaan ang diwa ng kalikasan ng pagiging makatao. Maaaring mawala o maubos ang mga kayamanan ng isang tao, at maging ang kanyang pagiging makabayan, subalit hindi ang panitikan. Sa panlipunan, pambansa, at pandaigdigang kaukulan, isa ang panitikan sa pinagbabatayan ng pagkakaroon ng buhay at katagumpayan ng isang bansa (De Juan, 2015). Salamin din ang panitikan sa kulturang gumagana sa isang komunidad. Sa bayan ng Matalom, bawat barangay ay mayaman sa tradisyon at kultura.

\section{Metodolohiya}

Ang pag-aaral na ito ay gumagamit ng kwalitatibong paraan ng pananaliksik sa anyong pagsusuri sa nilalaman (content analysis) at anyong historikal. Ang pagsusuring pangnilalamang ito ay ginamit sa pagsusuri ng bawat salita o pahayag na napapaloob sa bawat alamat ng Matalom, Leyte at ang pagtukoy sa lumilitaw na tema na siyang magpapakilala sa naiibang kultura at tradisyon ng bayan. Ang anyong historikal naman ayon kay Sauco (2011) ay sumasaklaw sa nakalipas. Binabakas dito ang pagkakasunod-sunod ng mga pangyayari, ang pag-unlad, ang mga dahilan ng mga bagay, ang sanhi at bunga. Batay sa mga datos at ebidensya, pinalalalim ang pag-unawa sa nakaraan kung paano at bakit nangyari ang mga bagay-bagay maging nag pinagmulan nito. Ang pamamaraang ito ang ginamit sa pagsusuri ng mga alamat sa mga piling barangay sa lungsod ng Matalom, Leyte dahil angkop itong gamitin sa pag-aaral upang magkaroon ng malalimang paghimay-himay sa mga napapaloob na kultura at tradisyon at mailarawan ang mga tema na nilalaman sa mga alamat.

\section{Resulta at Diskusyon}

Ang mga alamat sa bayan ng Matalom, Leyte ay napapalooban ng apat (4) na natatanging tema, ito ay ang (1) mabuting pakikipagkapwa-tao; (2) umaasa sa biyaya ng kalikasan; (3)pwersang ispirituwal at pangkalikasan; at (4) pinagsamang makalumang paniniwala at paniniwalang Kristiyano. 


\subsection{Mabuting Pakikipagkapwa-tao}

Ang pakikipagkapwa ay ang paraan ng pakikisalamuha sa ibang tao (at sa ibang nilalang) nang may buong paggalang at walang halong pagkukunwari (Enriquez, 2007). Sinabi naman ni (Meneses, 2018) na isang napakagandang katangian ng mga Pilipino ang mabuting pakikipagkapwa. Likas sa isang Pilipino ang mapanatili ang magandang pakikipag-ugnayan, ang umunawa at magbigay hindi lamang sa pamilya at mga kaibigan kundi maging sa mga higit na nangangailangan. Kilala siya sa kanyang katangiang mapagbigay, mapagmahal at mapaglingkod sa kapwa. Ito ay tatak niya, kaiba sa ibang lahi.

Likas na sa mga tao sa Matalom ang mabuting pakikipagkapwa. Sila ay matulungin, maawain, mapagkawang-gawa, mahusay makisama at masayahin. Simula pagkamulat ng mananaliksik sa lungsod ng Matalom ay wala pa siyang narinig na kaguluhan sa lugar patunay na ang mga mamamayan dito ay may mabuting pakikipagkapwa na isa sa pinakamagandang katangian ng tao.

Inilahad sa alamat ng barangay Caridad na:

“...likas na sa pagiging matulungin at mapagkawang-gawa ang mga taong nakatira sa barangay..."

Makikita naman sa alamat ng barangay Agbanga, San Salvador, Atltavista, Templanza at San Juan ang ganitong pahayag

“... nagtutulungan sa oras ng pangangailangan...”

Ibig sabihin nito kaya maayos ang relasyon o ugnayan ng bawat tao sa bayan mapa-upland, lowland at coastal ay sa kadahilanang palagi nagkakaisa at nagtutulungan ang mga tao sa lahat ng oras at mahusay makisama. Masayahin din ang mga tao sa lugar at bagaman sila ay abala sa paghahanapbuhay ay nagkakaroon pa rin sila ng oras para makapaglibang at makipagsalamuha sa ibang tao. Ilan sa mga barangay sa Matalom ang nagsasagawa ng “ensayo”. Ito ay pagtatanghal sa ibabaw ng entablado ng mga mahusay gumanap ng isang karakter tulad ng napakikinggan natin sa radio at napapanood sa telebisyon. Nauso ang "ensayo" noong unang panahon na kung saan aliw na aliw ang mga tao. Sa kasalukuyan, nalilibang ang mga tao tuwing magsasagawa ang barangay ng "bayli" noon na disco na ngayon. Tuwing sasapit ang kapistaha ay magkakaroon ng bayli sa gabi at sabong naman sa araw at iba pang mga palaro sa barangay na kinaaaliwan ng lahat.

Ngunit dahil sa pagbabago ng panahon at sa pag-unlad ng teknolohiya, ang 'bayli' noon na hindi maaaring sumayaw kapag walang kapareha ay nagbago na. Marahil napagtanto ng mga tao lalo na ng mga kabataan na gusto talaga nilang sumaya at maglibang kaya kahit walang kapareha ay sumasayaw na sila kapag may disco o kaya'y kahit baba-babae o lalaki-lalaki (pagtukoy sa ikatlong kasarian) ang sumayaw ay tinatanggap na.

Matutunghayan sa alamat ng barangay Templanza na:

"Dahil sa modernong panahon ngayon, ang lahat ay pwedi nang sumayaw maging ang mga babae o dalaga ay maari ng mamili ng kanilang makakapareha sa pagsasayaw”.

May pagbabago mang naganap sa kultura ang mga tao sa Matalom ay nanatili pa rin ang kanilang magandang ugnayan sa isa't isa.

\subsection{Tema 2. Umaasa sa Biyaya ng Kalikasan}

Ang Pilipinas ay mayaman sa mga likas na yaman kaya nga magpahanggang ngayon ang mga tao ay umaasa pa rin sa mga biyayang makukuha rito. Ang likas na yaman ay tumutukoy sa mga bagay na makukuha natin mula sa kalikasan tulad ng yamang lupa (mga bagay na itinanim o kusang nakukuha natin sa paligi); yamang tubig (mga bagay o pagkaing nakukuha natin sa ilog, sapa at dagat at iba pang anyo ng tubig); yamang mineral (mga bagay na namimina at nakukuha sa ilalim ng lupa); at yamang-gubat (mga bagay o hayop na

136 Consortia Academia Publishing (A partner of Network of Professional Researchers and Educators) 
makukuha natin sa kagubatan). Hindi ito gawa ng tao ngunit gawa ng Panginoon (Geonzon, 2016) ngunit kapag nagtatanim at nag-aalaga ang mga tao ay maaring likha niya ang nakuhang likas na yaman tulad ng palay, mais, saging at iba pa.

Isa sa pangunahing pinagkukunan ng ikinabubuhay ng mga tao sa lungsod ng Matalom ay ang ang likas na yaman at agrikultura. Karamihan dito ay nagsasaka at nangingisda. Ang mga mamayan sa upland at lowland ay nagsasaka, nagtutuba at nag-aabaka samantalang sa coastal, karamihan ay nangingisda dahil malapit sila sa dagat.

Ang Matalom, Leyte ay nahahati sa tatlong bahagi, ang coastal o malapit sa dagat, lowland o kapatagan at upland $\mathrm{o}$ bulubunduking bahagi ng Matalom, Leyte. Ang karagatan ng Matalom at ang karatig lungsod nito, ang Bato, Leyte ay mayaman sa mga yamang dagat kaya karamihan sa mga mamamayan ng lugar ay pangingisda, pangunguha at pag-aalaga ng mga kinhason o lamang dagat at maging ng mga sugpo ang pangunahing hanapbuhay . Sikat ang Matalom sa pagkakaroon ng iba't ibang lamang dagat kaya nga may mga negosyanteng nagpatayo rito ng mga natatanging kainan na nagluluto ng mga preskong isda, sugpo, alimango at iba pang lamang-dagat tulad ng Kiosko Food Park, Pagatpat at Superball. Makikita rin sa gilid ng malaking kalsada lalo na sa may bahagi ng Barangay Caridad hanggang Barangay Agbanga ang mga nagtitinda ng mga preskong isda, iba’t ibang klase ng kinhason at sugpo.

Sa lowland naman ay makikita ang mga palayan, may mga gulayan at mga taong nagtutuba at nagkokpra dahil maraming puno ng niyog ang tumutubo sa lugar samantalang sa upland naman ay karaniwang makikita ang nag-aabaka, nagtutuba at nagkokopra. Marami rin ang nagsasaka sa lugar tulad ng sa lowland.

Mababasa sa alamat ng barangay Punong, barangay San Pedro, barangay Caridad, at barangay Agbanga ang mga pahayag na nagpapatunay na umaasa pa rin sila sa mga likas na yaman:,

"Ang lugar na ito kahit magubat ay sagana sa tubig. Marami kasing sapang dumadaloy kung saan-saan na may malaking pakinabang sa kanila sapagkat dito sila kumukuha ng tubig na magagamit sa pang-araw-araw, dito sila naliligo at naglalaba at nakakakuha rin sila rito ng mga yamang-tubig tulad ng isda, suso at alwang o talangka..."

"At dahil malapit sa dagat ang barangay Punong kaya pangunahin ding hanapbuhay ng mga tao ang pangingisda..."

"Karaniwang hanapbuhay ng mga tao sa barangay San Pedro ay pagtitinda, pagtutuba, paghahabi ng abaka, at pangingisda..."

"Karaniwang hanapbuhay sa Barangay Caridad ay pangingisda at pagnenegosyo..."

"Karaniwan sa hanapbuhay ng mga tao sa Agbanga ay pagtatanim ng palay, pangingisda at pangunguha ng mga yamang dagat at ibinibenta..."

Sinabi ni Atanacio (2013) na napaliligiran ng mga katubigang sagana sa mga yamang-dagat ang mga pulo ng RehiyonVIII. Malaki ang naitutulong nito sa kabuhayan ng mga Waray at Bisaya (tawag sa mga taong nakatirasa Samar at Leyte).

Ayon naman kay Honradez (2016) ang kapaligiran ay may kinalaman sa gawain ng tao sa isang lugar lalo't higit sa hanapbuhay o pinagkakakitaan ng mga naninirahan dito. Kaya nga kadalasan sa mga taong nakatira sa coastal ay pangingisda at pangunguha ng mga yamang dagat ang pangunahing ikinabubuhay ng mga tao.Ganun din ang sinabi ni Peña (2015) na ang hanapbuhay at pamumuhay ng mga tao ay maiuugnay sa kapaligirang mayroon sila.

Maliban sa pangingisda at pagtitinda sa mga nakuhang yamang dagat ay may mga nagsaka rin kahit malapit sila sa coastal. Ginamit nila ang mga lupaing nasa bahaging papuntang kapatagan para tamnan ng mga palay, 
mais at kamote. Kadalasan sa produkto nila tulad ng mais, kamote at saging ay ginamit lamang sa pansariling konsumo dahil hindi naman gaanong malawak ang laupaing pinagtamnan maliban sa bahaging Itum na may malawak na palayan na ang ibang ani ay ipinagbibili dahil sobra $\mathrm{n}$ sa pansariling konsumo.

Sa kapatagan naman maging sa kabundukan o upland na bahagi ng Matalom, karaniwan ay nagsasaka. Mababasa sa mga alamat ang karaniwang hanapbuhay o ikinabubuhay ng mga tao pagtatanim ng palay, mais, kamote at iba pang yamang lupa. Mayroon ding gumagawa ng sinamay at pagtutuba.

"Karaniwan kasi sa hanapbuhay ng mga tao sa lugar ng Pong-on ay pagsasaka, paggawa ng sinamay, at paghahabi ng abaka..."

"Noong unang panahon, kaunti pa lamang ang nakatira sa Brgy. Taglibas. Ang unang mag-asawang nakatira rito ay sina Daniel at Oryang. Mayroon silang limang anak. Kilala ang mag-asawa sa pagiging masipag. Ang kanilang hanapbuhay ay ang pagkakaingin na ginagamitan ng bolo para maputol ang mga punongkahoy at pagkatapos sinusunog ang mga natirang damo at tsaka tamnan ng iba't ibang pananim. Bagaman pagtutuba at pagsasaka pa rin ang pangunahing hanapbuhay ng mga tao sa Taglibas ay may nakapagpatayo na ng mga malalaking bahay..."

"Isa sa karaniwang hanapbuhay ng mga tao rito ay pagsasaka na kung saan tuwing matapos nilang gamitin ang kanilang kalabaw at pagpapahingahin na nila ito ay doon nila dinadala sa napakalaking puno ng tugas...”

"Dahil may kalayuan sa lungsod ang barangay Bagong lipunan, tanging pagsasaka at pagtutuba lang ang hanapbuhay ng mga tao sa lugar.”

Patunay lamang ang mga pahayag na ito na makikita sa mga alamat na ang mga mamamayan ng Matalom, Leyte ay umaasa pa rin hanggang ngayon sa mga likas na yamang makukuha sa dagat, sa lupa at sa kabundukan.

Ang Matalom, Leyte ay bahagi ng Rehiyon 8. Ang rehiyong ito ay mayaman sa mga produktong tulad ng palay, mais, niyog, tuba, abaka at iba pa. Ayon kay Atanacio (2013), pagsasaka ang pangunahing hanapbuhay ng taga-rehiyon VIII. Kabilang sa kanilang mahahalagang pananim ay ang palay, tubo, mais, niyog, abaka, tabako, gulay, at kape. Ang mga Matalomnon maging ang mga karatig lungsod ay masisipag sa pagsasaka kaya nga hindi na sila kailangang umangkat pa ng maraming produkto mula sa iba pang lugar.

\subsection{Tema 3. Pwersang Ispiritwal at Pangkalikasan}

Ang temang ito ay tumutukoy sa paniniwalang may mga nilalang na maaring nakikita o hindi nakikita na kayang magbigay ng anumang karamdaman o sakit sa isang tao na kung saan ginagamot ang karamdamang ito ng isang albularyo gamit ang mga halamang gamot at orasyon. Tumutukoy din ang temang ito na kailangang magsagawa ng ritwal bago gawin ang mga bagay tulad ng pagtatanim at pag-aani.

Ayon sa pananaliksik nina Delim, Dollega, at Pacheco at mga kasama (2016) likas na sa mga Pilipino ang pagiging mapagmahal sa kalikasan kung kaya't kapag may dumarating na hindi kanais-nais na pangyayari agad itong ikinonsidera na may koneksyon sa kapaligiran. Para sa mga taong ito, naniniwala sila na ang isang pangkaraniwang araw ay nababalot at hindi mabubuo kung walang mga paniniwalang sinusunod. Dagdag pa nila, kahit noon pa man ang mga ninuno ay may mga paniniwala nang sinunod na kagaya ng pag-aalay ng prutas o pagkain sa anito upang bantayan ang pananim at hindi pestihin o kaya ang pag-aalay ng panalangin sa pamamagitan ng ritwal na sayaw kay Bathala upang payabungin ang palay at pasaganahin ang ani nito.

Bagaman may mga pwersang nakatutulong sa pagkakaroon ng masaganang ani ay mayroon din namang mga nilalang na nakapagbibigay ng karamdaman sa mga tao. Sila ay pinaniniwalaang nagtataglay ng kung anong kapangyarihan. Maaring isang tao o kaya’y mga maligno. Ang mga karamdamang hatid nila sa tao ay tanging 
ang albularyo lang ang makapagpapagaling.

Ayon kay Yee (2018), bago dalhin sa manggagamot o doktor ang may sakit ginagamot muna ito ng isang albularyo o kaya'y sa pari para mabindisyunan. Kapag naghihingalo na ay tsaka pa lamang dadalhin sa doctor. Kaya naniniwala pa ang mga Matalomnon tungkol sa binisayang panggagamot ay dahil gumagaling umano ang mga may sakit na ginagamot sa albularyo.

Ang paniniwala sa mga kulam (barang), sa usog (bujag) at sa binisayang panggagamot ng albularyo ay nananatili pa sa Matalom, Leyte. Ang ganitong paraan ay isinasagawa sa barangay Caningag, barangay Itum, at barangay bagong Lipunan. Nagsasagawa naman ng mga ritwal sa masaganang ani ang mga taga barangay Taglibas, barangay Itum, barangay Caningag, barangay Punong, barangay bagong Lipunan at barangay Altavista. Ginagawa nila ang ritwal tuwing sila ay nagtatanim ng palay, mais, gulay, kamote at iba pang pananim. May ritwal din bago anihin ang mga palay. Sa coastal naman tulad ng barangay Punong ay nagsasagawa ang mangingisda ng ritwal bago mangisda.

Makikita sa nilalaman ng alamat na ang kalusugan ng tao ay nakabatay sa pwersang ispirituwal at pangkalikasan.

"Ang mga pumapalaot sa dagat para mangisda ay nagdadala pa noon ng abo at isasabog sa laot sabay bigkas ng orasyon para daw marami silang mahuhuling isda sa karagatan. Kapag bago naman ang lambat na gagamitin ay palinaan muna nila bago gamitin...”

"Sa tuwing aanihin na nila ang palay ay "magpalihi” muna sila. Kukuha muna sila nang kaunti tapos babayuhin para maging bigas at kapag naging bigas na ay sasaingin at lalagyan ng pandan para mabango. Nilalagyan rin nila ito ng kaunting asin..."

"Ang dahon ay ginamit na panggamot sa mga sakit tulad ng lagnat, ubo, pagtatae, pagsususuka at iba pa...”

"Naniniwala sila na kapag may nagkasakit ay kinulam (gibarang) at ginagamot ito ng isang albularyo. Ang albularyo ay bumibigkas ng orasyon sabay ihip (tayhop) ng hangin sa bahagi ng katawan na tinamaan ng sakit. Kapag may nagnakaw naman ay nahuhulaan umano ng isang "magtitigi" gamit ang barya. Naituturo daw ng barya ang salarin dahil kapag binanggit ang pangalan, ang barya ay tatayo at kapag hindi ang salarin ang nabanggit ang barya ay hndi gagalaw. Naniniwala rin ang mga tao sa lugar na ito sa mga nilalang na hindi nakikita ng ordinaryong tao na kung saan kapag silay ginalit ay mananakit din umano sa mga tao. At syempre pa ang albularyo na naman ang gagamot sa mga sakit na dumadapo sa kanila..."

Sa kasalukuyan, nakadependi na sa isang tao kung ano ang dapat nilang paniwalaan o gagawin. Kung susubukan nilang sundin at gawin ang mga ritwal at mga pamahiin ay wala namang mawawala sa kanila, ang mahalaga nakahanda silang harapin ang anumang maidudulot ng kanilang mga kilos.

\subsection{Tema 4. Pinagsamang Lumang Paniniwala at Paniniwalang Kristiyano}

Ang temang ito ay tumutukoy sa pagiging maka-Diyos at paniniwala sa mga diyos-dyosan o paniniwala sa mga engkanto at diwata. Likas na sa mga mamamayan ng Matalom, Leyte ang pagiging maka-Diyos. Makikita ang tradisyong ito sa pagsisimba nila tuwing lingo o tuwing may misa sa kapilya man o sa malaking simbahan ng Matalom. May ilang mag-anak pa din sa kasalukuyan ang nagdarasal tuwing sasapit ang ika alas-sais ng hapon at alas tres ng madaling araw. Tuwing sasapit naman ang mahal na araw o kaya'y kapistahan sa bawat barangay lalo pa kung kapistahan ng lungsod, ang pangkat ng mga tao na pinamunuan ng mga katikista at naglilingkod sa simbahan at sa kapilya ay nagsagawa ng prosisyon sa mga imahe na ibinahay-bahay. Nagdarasal sila at nananalangin sa bawat bahay na pinaglagyan ng kanilang mga patron. Nagdaraos din sila ng kapistahan taon-taon bilang pasasalamat sa kanilang patron. Ito ay patunay na ang mga Matalomnon ay maka-Diyos. 
Kung mapapansin natin karamihan sa mga Pilipino ay nagsisimba tuwing Linggo at nagdedebosyon. Sinabi ni Eugenio (2007) na ang pagiging maka-Diyos ng mga Pilipino ay isang natatanging pagkakakilanlan. Kilala ang mga Pilipino sa pagiging maka-Dios at may pagpapahalaga sa kanilang relihiyon. Binanggit ni Joven (2013) sa kanyang tesis na ang paniniwala o pananampalataya ay bahagi ng tao sa kanilang mga gawain araw-araw. Nakaugalian na ng mga Pilipino ang pagiging relihiyoso o maka-Diyos. Ayon kay Abangan (2009) ang mga Pilipino ay maka-Diyos sapagkat buong pamilya ang sama-samang nagsisimba, nagrorosaryo at nagdarasal tuwing alas sais o orasyon. Ang Dios ang siyang sandigan ng mga Pilipino sa oras ng mga problema at pangangailangan. Hindi nito nakakalimutang tumawag sa Maykapal at magpasalamat sa mga biyayang natatanggap.

Makikita sa mga alamat ang pagiging relihiyoso ng mga tao sa lungsod ng Matalom, Leyte. Bahagi na ng kulturang Pilipino ang pagsamba tuwing linggo at pag-oorasyon. Ipinahayag nga sa blog ng Understanding our Filipino Value System na isa sa mga kaugalian ng mga Pilipino ay ang paniniwala sa Panginoon at pagsamba nito.

Mababasa sa mga alamat na ang mga tao sa Matalom ay relihiyoso at maka-Diyos:

"Ang imahe ng Santo ay palaging ipinoprosisyon sa kabahayan hanggang sa iuwi na nanaman sa simbahan. Ang mga tao rito ay mga relihiyoso at palaging nadsisismba at nagdarasal..."

"Ang mga mangingisda bago lumayag sa laot ay taimtim munang nagdarasalpara gabayan sila at bigyan ng mas maraming biyaya mula sa dagat..."

"Pinalitan ang pangalang Tiyanggeng Daan ng San Pedro na kung saan ito rin ang kanilang patron dahil ayon sa kanilang paniniwala ay si San Pedro raw ang gumagabay sa kanila sa laot tuwing nangingisda sila. Ang mga tao rito ay mga relihiyoso at mahigpit ang pananampalataya sa Dios. Tuwing ika-alas $6 \mathrm{ng}$ hapon at ika-3 ng madaling-araw, ang bawat miyembro $\mathrm{ng}$ pamilya ay nagdarasal. Hanggang ngayon ay relihiyoso pa rin ang mga tao rito ngunit bihira nalang ang mga nagdarasal o nagrorosaryo tuwing sasapit ang alas sai $n g$ hapon at alas tres ng madaling-aral..."

"Tuwing sasapit naman ang ika-6 ng hapon ay magtitipon-tipon na ang mga miyembro ng pamilya para magdasal; Idinaraos ang kapistahan sa lugar tuwing sasapit ang ika-16 ng Agosto kada taon at ang mga tao ay nagsipaghanada bilang pasasalamat sa kanilang patron..."

"Silang dalawa ay mga inosente ngunit mababait at may takot sa Diyos..."

“Naniniwala ang mga tao sa lugar na bawat kahilingan nila'y ipinagkakaloob ng imahe. Dahil dito palagi ng nagsisimba ang mga tao at labis ang papanampalataya nila na milagroso ang imahe. At nang dumating na ang panahong maaari ng maging barangay ang lugar ay pinalitan ng mga tao ang pangalang Pong-on ng Barangay San Salvador bilang pasasalamat sa kabutihang ipinagkaloob ng imahe sa kanilang lugar. Nagdaraos ng kapistahan ang barangay San Salvador tuwing sasapit ang ika-10 ng Enero at bawat tahanan ay masayang naghahandaan...”

"Ang dahon naman nito ay mabango na kung saan tuwing may kasal ginagamit ito bilang palamuti at inaalay sa Mahal na Birhen tuwing Flores de Mayo..."

"Tuwang-tuwa ang lahat at bilang pasasalamat ay ipinangalan nila ang lugar sa kanilang patron na si San Juan...”

"Nagdaraos ng kapistahan ang lugar tuwing sasapit ang ika-5 ng Abril at bahagi ng pagdiriwang ang pagkakaroon ng pagtatanghal, sabong at bayli sa gabi. Nagkakaroon din sila ng koronasyon bilang "fund raising" na makatutulong sa paglinang ng kanilang barangay. 
Kapag may gawain ay nagtutulungan ang mga mamamayan lalo na kung ito ay may kinalaman sa kanilang barangay. Nagtitipon-tipon (assemble) naman sila tuwing Disyembre. Silay nag-aaurora sa kanilang patron na si Sr. San Vicente Ferer. Tuwing simbang gabi, bawat pamilya ay nagdadala ng mga pagkain sa kapilya o bahay dasalan na pinagsasaluhan ng lahat ng nagsipagsimba. Isinasagawa ito hanggang Disyembre 24."

Bagman maka-Diyos at makarelihiyon ang mga tao sa Matalom ay hindi pa rin maiiwasan ang paniniwala nila sa mga diyos-diyosan o paniniwala sa mga engkanto, duwendi at iba pang mga lamang lupa na hindi nakikita ng ordinaryong mga mata.

Sa Mitolohiyang Pilipino, ang Diwata ay isang katauhang katulad ng mga engkanto (fairies) o nimpa (nymph) na naninirahan sa mga puno na tagapagbantay ng ispiritu ng kalikasan na nagdadala ng pagpapala o sumpa sa mga taong nagbibigay ng benipisyo o pinsala sa mga gubat at mga bundok (Villan, 2013). Ayon kay Entico (2019) may babae at lalaking engkanto. Engkantada o diwata ang tawag sa babae at engkantado naman ang tawag sa mga lalaki. Hindi pangkaraniwan ang kanilang ganda, magandang mukha, maputi o maputla ang kanilang balat. Malaginto ang mahahabang buhok, asul na mga mata at mas matangkad kumpara sa mga pangkaraniwang tao.

Ang diwata ay mga nilalang na nagbabantay sa ating kalikasan na mailap sa kabila ng angkin nilang kagandahan. Hindi malapit sa tao ang mga ito. Katunayan ayaw daw nilang ginagambala ang kanilang tirahan. At kapag sila'y nagalit, buhay daw ang kapalit. (GMA News Online, 2013). Kaya nga ang ilan sa mga tao noon sa Matalom lalo na ng mga matatanda ay nag-alay ng mga hayop o pagkain sa gubat dahil natatakot silang masaktan sa mga nilalang na ito.

Inilahad sa alamat ng barangay Agbanga na:

“...Kaunti pa lamang ang mga nakatira rito noon at ang mga tao ay naniniwala pa at sumasamba sa kinilalang Diyos ng kagubatan na kung saan nagdadala sila ng kanilang mga alay tuwing Biyernes..."

May ganito ring paniniwala ang mga taga-arangay President Garcia, barangay barangay San Juan at Bagong lipunan.

"Noon may mga naniwala pa sa mga diwata na kailangang pag-alayan ng mga hayop para hindi gagalawin ng ma ito ang mga tao ngunit ngayon ay wala na dahil din daw sa pagkawala ng gubat na tinitirhan ng mga ito..."

"Lumapit sila sa isang albularyo sa lugar at sinabing kailangan nilang mag-alay ng mga hayop sa mga diwata upang pahintulutan silang makapagtayo ng tulay. Sinira daw kasi ng mga tao ang tirahan ng mga diwata..."

"Mapuno rin ang lugar na ito noon kaya hindi maiwasan na ang mga tao ay maniwala sa mga nilalang na hindi nakikita ng orninaryong mga mata. Ang iba ay nag-aalay pa noon ng pagkain at dinadala sa masusukal na bahagi lalo na kapag may kinakasal o may handaan, inaalok din nila ng pagkain ang mga engkanto ngunit hindi nila nilalagyan ng asin. Ayaw dw kasi ng mga engkanto ng mga pagkaing may asin..."

Sa kasalukuyan, bagaman naniniwala pa rin ang mga tao sa mga nilalang tulad ng mga engkanto ay hindi na sila sumasamba rito at nag-aalay ng kung ano-ano katulad ng ginawa ng mga matatanda noon. Umiiwas nalang sila rito para hindi sila masaktan tulad ng pagsasabi ng "Tabi, tabi!" tuwing mapadaan ang mga tao sa magugubat na bahagi o sa malalaking puno o kaya'y sa ilog na pinaniwalaang tirahan ng mga diwata at engkantado. Iisang Diyos nalang ang sinasamba ng mga tao ngayon sa Matalom, Leyte. Ito ang Diyos na makapangyarihan sa lahat at siyang may likha sa sanlibutan. 
Halos ang mga baranggay na matatagpuan sa bayan ng Matalom, Leyte ay magkadikit kung gayon mas nagkakaroon ng manipestasyon na ang nakagawiang kultura at tradisyon sa partikular na lokasyon ay madaling maipasa dahil sa transmisyon ng mga tao sa araw-araw ng kanilang pamumuhay at gawi. Ayon kay Falae (2018), ang kultura ay isang kolektibong itinuturing manifestation ng intelektwal na kakayahan ng tao at ang tradisyon naman ay isang pag-uugali o paniniwala na may natatanging kahulugan na ipinasa pababa sa loob ng isang lipunan o pangkat mula sa isang henerasyon patungo sa isa pa. Kaugnay nito sinabi ni Justin (2014), ang kulturang Pilipino ay pinaghalong impluwensiya ng mga katutubong tradisyon at mga kultura ng mga unang mangangalakal at mananakop nito noon.

Katulad ng Matalom, Leyte na nasa upland, lowland at coastal na bahagi, may kinikilala ang bawat lokasyon sa kanilang sarili o higit pa na mga salik katulad ng lipi, wika, relihiyon o sa isang magkatulad na kasaysayan na makikita sa kanilang panitikan lalong-lalo na sa alamat. Ang bayan ng Matalom, Leyte ay mayaman sa kultura at tradisyon na dapat na linangin at ipakilala sa mga bagong henerasyon upang mabuhay at magamit sa kasalukuyan. Patunay na ang pagbuhay muli sa natutulog na kultura at tradisyon ay siyang mamumulaklak na magdadala sa isang lugar sa kariktan nitong katayuan.

\section{Konklusyon}

Ang mga alamat ng bayan ng Matalom, Leyte ay isang manipestasyon na kakikitaan ng mayamang kultura at tradisyon ang bayan na nagbibigay-diin sa kahalagahang pantao at paniniwala ng mga taga-Matalomnon. Isinasalamin ng bawat alamat sa piling baranggay sa bayan ng Matalom, Leyte ang pangkalahatang diwa, pananaw, kaugalian at adhikain ng mga tao sa pagtalima sa mga paniniwala sa kanilang lipunan at kapaligiran. Ang pag-aaral na ito ay nagpapakita sa alamat bilang larawan sa identidad ng Matalomnon. Lente ang mga akdang ito sa buhay na karanasan ng mga tao sa kasalukuyan. Ang resulta ng pag-aaral na ito sa mga alamat ay maging instrumento bilang lunsaran sa pagtuturo at pagkatuto sa panitikan ng lugar na siyang tugon sa kontekstuwalisasyon at lokalisasyon $\mathrm{ng} \mathrm{K}$ to 12 kurikulum. Daan din ito upang manumbalik sa mga Matalomnong mag-aaral ang makulay na kwento ng kanilang lugar.

\section{Mga Sanggunian}

Abangan, J. (2009). Literary analysis of twenty-five legends of Negros Oriental [Mga sinuring alamat ng dalawampu't limang bayan ng Negros Oriental]. Di Nailathalang Tesis. Cebu Normal University, Cebu City, Philippines.

Atanacio, N. (2013). Region VIII Eastern Visayas [Rehiyon VIII Silangang Visayas]. Retrieved from https://www.scribd.com/doc/181414427/rehiyon-viii-silangang-visayas-docx

De Juan (2013). The legend and its brief history [Ang alamat at ang maikling kasaysayan nito]. Retrieved from https://filipinotek.wordpress.com/2013/03/29/ang-alamat-at-ang-maikling -kasaysayan-nito.

Delim, J., et al. (2016). A research on superstitiuous belief [Isang pananaliksik tungkol sa pamahiin na pinaniniwalaan]. Retrieved from https://prezi.com/o8emprgbxzix/isang-pananaliksik-tungkol-sa-pamahiin-na-pinaniniwalaan/

DepEd (2015). Filipino curriculum guide baitang 1-10. Retrieved from www.deped.gov.ph/site/default/files/page/2016/Filipino\%20CG.pdf

Du, N. (2014). Understanding the ancient non-material cultures of B'laan of Brgy. Pisan, Kabacan, Cotabato from their folktale [Pagdalumat sa mga sinaunag di-materyal na kultura ng mga B'laan sa Brgy. Pisan, Kabacan, Cotabato mula sa kanilang kwentong bayan]. Asia Pacific Journal of Multidisciplinary Research, 2(6), 158-163.

Enriquez, V. G. (2007). Kapwa: A core concept in Filipino socialpsychology. In A. Navarro \& F. Bolante (Eds.), Mga babasahin sa Agham Panlipunang Pilipino: Sikolohiyang Pilipino, pilipinohiya, at pantayong pananaw. Manila: C \& E Publishing

Entico, J. (2019). Where did the fairies come from? [Saan nagmula ang mga diwata?] Retrieved from http://www.mangkukulam.com/kababalaghan/363/Engkanto

142 Consortia Academia Publishing (A partner of Network of Professional Researchers and Educators) 
Pagdukal sa mga alamat ng mga barangay sa Bayan ng Matalom, Leyte: Isang suring-tematiko

Eugenio, D. (2007). Philipinnes folk literature. The legends. Quezon City: UP Press.

Falae, K. (2018). The legend and its importance [Ang alamat at kahalagahan nito] Retrieved from http://www.suriblogspot.com/2014/07/ang-alamat-at-kahalagahan-nito.html

Geonzon, E. (2016). Natural resources [Mga likas na yaman]. Retrieved from https:// www.slideshare.net/elerageonzon/mga-likas-na-yaman-67240648

GMA News Online, (2013). Does John still believes in fairy? [Naniniwala pa ba sa diwata si Juan?] Retrieved from

https://www.gmanetwork.com/news/newstv/ijuander/317550/i-juander-naniniwala-pa-ba-sa-diwata-si-j uan/story/

Honradez, E. (2016). Linking environment and type of occupation [Pag-uugnay ng kapaligiran at uri ng hanapbuhay]. Retrieved from

https://www.slideshare.net/edithahonradez/yunit-2-aralin-1-pag-uugnay-ng-kapaligiran-at-uri-ng-hanap buhay

Joven, M. (2013). Analysis of Filipino thesis of selected SUC's of region VII [Pagusuri sa mga Filipino tesis ng mga piling SUC's ng rehiyon VII]. Di-nalathalang tesis. Southern Leyte State University Tomas Oppus, Southern Leyte.

Justin, L. (2014). The importance of legend in the Philippines [Kahalagahan ng Alamat sa Pilipinas]. Retrieved from http://www.suriblogspot.com/2014/07/kahalagahan-ng-kaalaman-sa-alamat.html

Lamiseria, G. (2016). Live-out Seminar-Workshop on Contextualization, Localization and Indigenization of Filipino Curriculum. Palo, Tacloban, Leyte, Philippines

Meneses, K. (2018). Pakikipagkapwa: A Filipino value in attempt to counter biodiversity and cultural diversity loss. Solidarity: The Journal of Catholic Social Thought and Secular Ethics, 8(1).

Menor, A. (2015). Culture: Studies and perspective on Filipino subject [Kultura: Pag-aaral at pananaw sa asignaturang Pilipino]. Retrieved from https://thedailyguardian.netcommunity-news/kultura-pag-aaral-at-pananaw-sa-asignaturang-filipino/

Panganiban, J. V., \& Casanova, A. P. (2005). The Philippine literatures [Mga panitikan sa Pilipinas]. Retrieved from http://worldcat.org.ph/

Peña, R. (2015). Introduction to the poems of the copra in the Bondoc Peninsula towards the investigation of the social ego [Panimulang dalumat sa mga tula ng mga magkokopra sa Bondoc Peninsula tungo sa pagsiyasat sa panlipunang kaakuhan]. Bisig Journal: Manggagawa at Paggawa sa Panahon ng Neoliberalismo, 2.

Ramos, M. S. (1984). Filipino literature [Panitikang Pilipino]. Manila: Katha Publishing Company.

Sauco, C. (2011). Philippine regional literature [Panitikang panrehiyon ng Pilipinas]. Goodwill Trading Co.

Villafuerte, P. V. (2000). Regional literature in the Philippines [Panitikang panrehiyon sa Pilipinas]. Valenzuela City: Mutya Publishing House.

Villan, D. (2013). Over there and creatures of wonder [Sa dako pa roon at mga nilalang ng kababalaghan]. Retrieved from http://sadakoparoon.blogspot.com/2013/02/diwata.html

Yee, A. (2018). Albularyo, midwife and sorcerer [Albularyo, hilot at mangkukulam]. Retrieved from https://www.ohmybuhay.com/albularyo-and-mangkukulam/\#.XMOBtYlKjMx

Young, J. (2007). Philippine Myths and Legends. Retrieved from http:///philippinemythsandlegends.ph 
Gili, V. G., \& Galdo, M. B. 